\title{
AFM/STM Modification of Thin Sb Films on 6H-SiC(0001)
}

\author{
P. Mazur, S. Zuber, M. Grodzicki* And A. Ciszewski \\ Institute of Experimental Physics, University of Wrocław, pl. M. Borna 9, 50-204 Wrocław, Poland \\ Atomic force microscopy and scanning tunneling microscopy have been used for nanometer scale modifications \\ of $\mathrm{Sb}$ films deposited on $6 \mathrm{H}-\mathrm{SiC}(0001)$ surface. The films are grown in situ by vapor deposition under ultrahigh \\ vacuum. The growth follows the Volmer-Weber mode. Ultrathin (up to $3 \mathrm{~nm}$ on average) Sb films which consist \\ of no coalesced islands can be modified by moving the island over the substrate surface by the AFM tip. Thicker \\ films, which are firmer due to the coalescence, can be modified by the field and/or current produced by STM tip. \\ Final result of the modifications is adsorbate free $6 \mathrm{H}-\mathrm{SiC}(0001)$ surface restitution over the modified area.
}

DOI: 10.12693/APhysPolA.126.1131

PACS: 81.05.Dz, 68.37.Ps, 68.37.Ef, 68.37.-d, 81.10.Bk, 81.07.-b

\section{Introduction}

Scanning probe microscopies (SPM) are useful tools not only for imaging the surfaces but also for its intended processing and modification [1]. In surface engineering, the SPM techniques enable scratching of the surface and modifying it by current or electric field $[2,3]$. Such controlled thin film alteration allows the SPM techniques to be employed in lithography - in this particular case called scanning probe lithography (SPL) [4]. The SPL is currently developing rapidly and steady progress of the technique is observed. Researchers are looking for suitable systems of layered materials from which the deposit could be easily removed or modified and it is one of the up-to-date objectives in the field of nanotechnology.

$\mathrm{The} \mathrm{Sb} / \mathrm{SiC}(0001)$ system is promising for such applications. Silicon carbide is suitable as a substrate because of its well defined and easy to be controlled surface. The material is hard and highly chemically resistant, thus should not be subject to modifications [5]. Furthermore, $\mathrm{SiC}$ is a wide energy gap semiconductor widely used in electronics, in particular it is very attractive to applications in opto-, high-frequency, and high power microelectronics due to its unique properties such as large critical breakdown electric field, high-temperature stability and high thermal conductivity [6]. Antimony is also an important material in electronics and optoelectronics technologies. It is resistant to acids, does not react with oxygen and remains stable in air; has a good thermal conductivity. It is widely used as surfactant for better control of elementary growth processes of III-V semiconductors [7]. Its medium melting point permits the material to be easy evaporated from the substrate surface under ultrahigh vacuum (UHV) conditions.

In the present study, atomic force microscopy (AFM) working in contact mode and scanning tunneling microscopy (STM) are used for inspection of the properties

\footnotetext{
${ }^{*}$ corresponding author; e-mail: milosz.grodzicki@ifd.uni.wroc.pl
}

of thin $\mathrm{Sb}$ films grown on $6 \mathrm{H}-\mathrm{SiC}(0001)$ surface and as a tool for its topography and morphology alterations at nanometer scale.

\section{Experimental details}

Samples, around $8 \times 4 \mathrm{~mm}^{2}$ in size, cut out from $6 \mathrm{H}-$ $\mathrm{SiC}(0001) 3{ }^{\circ} \mathrm{C}$ off-axis wafers were used in this study. They were covered by atomically flat $n$-type (nitrogendoped) Si-face of epitaxial deposited $\mathrm{SiC}$ layer, which was used as a substrate. Prior to the measurements the substrates were ex situ degreased in acetone, next dipped in $\mathrm{HF}$ and washed in distilled water, and dried in air. UHV surface analysis systems housing the VT STM/AFM head (Omicron) with the base pressure $\approx 1 \times 10^{-10}$ Torr were used. Before $\mathrm{Sb}$ deposition (99.999), the substrate was heated for several min at $950 \mathrm{~K}$ under UHV conditions in order to remove surface contamination. Heating was carried out by radiation from a heater non-contacting the sample. Temperature of the sample was measured with a thermocouple (type $\mathrm{K}$ ). The $\mathrm{Sb}$ films were deposited from an electron beam evaporator under operating pressure lower than $1 \times 10^{-9}$ Torr. Average Sb-film thickness was quantified by a quartz microbalance. AFM was operated in the contact mode with the tip's force constant $0.12 \mathrm{~N} / \mathrm{m}$. STM images were recorded in constant current mode with bias voltages from 0.5 to $5 \mathrm{~V}$ at tunneling currents ranging from 0.8 to $1.5 \mathrm{nA}$. Electrochemically etched tungsten wires were used as the STM tips. The images were analyzed using the WSxM software [8]. All measurements were done at room temperature.

\section{Results and discussion}

AFM/STM analysis of the $\mathrm{SiC}(0001)$ surface topography shows the presence of terraces whose width ranges from 180 to $310 \mathrm{~nm}$, and height from 5 to $15 \mathrm{~nm}$. Most of the terraces are $\approx 265 \mathrm{~nm}$ in width and $\approx 15 \mathrm{~nm}$ in height (which height corresponds to ten lattice parameters $c$ of $6 \mathrm{H}-\mathrm{SiC}(0001))$. The deposition of $\mathrm{Sb}$ on $\mathrm{SiC}(0001)$ follows the Volmer-Weber growth mode. Beginning from 
earliest growth stages the adsorbate forms uniformly distributed island. Their population and dimensions grow with the increase of average amount of the deposit.

AFM studies reveal that the very first nuclei of the $\mathrm{Sb}$ islands are very weakly bound to the substrate and could be moved easily over the surface by AFM tip. They can be pulled or pushed by the tip. Repeated scanning over the same area results in sweeping off the adsorbate. Successive stages of the sweeping are shown in Fig. 1. In this case the deposit of average thickness of about $0.5 \mathrm{~nm}$ has been gradually pulled or pushed out from the scanned region after each subsequent scan. The most amount of $\mathrm{Sb}$ has been removed from the scanned surface at the beginning of the procedure during the first and second scan. The $\mathrm{SiC}(0001)$ surface has been cleaned of the deposit after the last sixth scan.

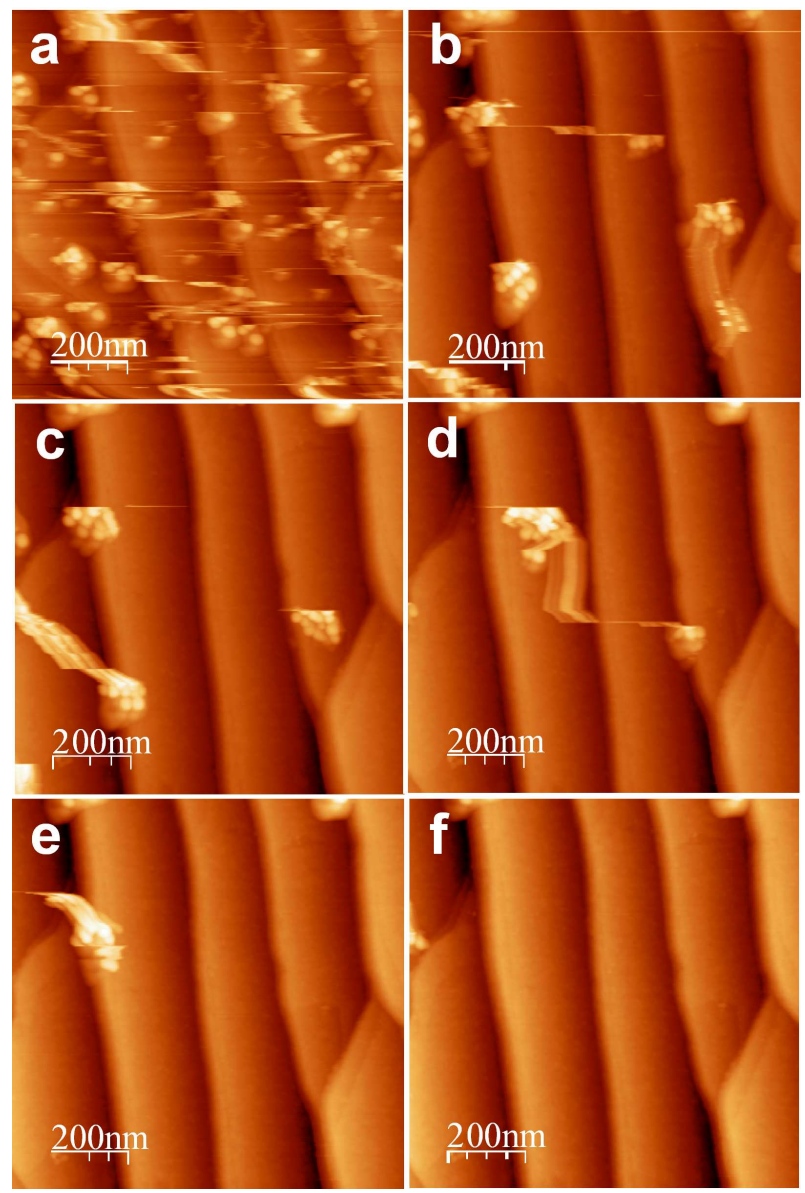

Fig. 1. The sequence of AFM images taken after each of six scans (a-f) over $\mathrm{Sb}$ film of mean thickness $0.5 \mathrm{~nm} ; \mathrm{Sb}$ is gradually pulled or pushed out from the scanned region after each subsequent scan; scanned area: $1 \times 1 \mu \mathrm{m}^{2}$. The 6 th scan results in complete removal of the deposit. Range of the color scale amounts from 0 to $30 \mathrm{~nm}$.

Gradual increase of the amount of Sb on the surface, following the deposition, increases and merges the nuclei into 3D islands. The islands are stronger bound to the surface but they also can be manipulated by the AFM tip. If the cantilever load is not efficient, scanning in this case leads to $\mathrm{Sb}$ accumulation at terrace edges, which is illustrated in Fig. 2. The adsorbate accumulates stacking up to about $10 \mathrm{~nm}$ above the terrace surface. The result of repeated scanning by the AFM tip over selected region of the substrate for the purpose of Sb removal is shown in Fig. 3. The image covers two areas, the one that is "brushed down" of Sb and another one still covered with $\mathrm{Sb}$. A border between these two regions is clearly visible. The area covered by the adsorbate cannot be imaged well because the AFM tip drags the adsorbate instead of following its real topography. It is confirmed by the deflection mapping (see Fig. 4b).

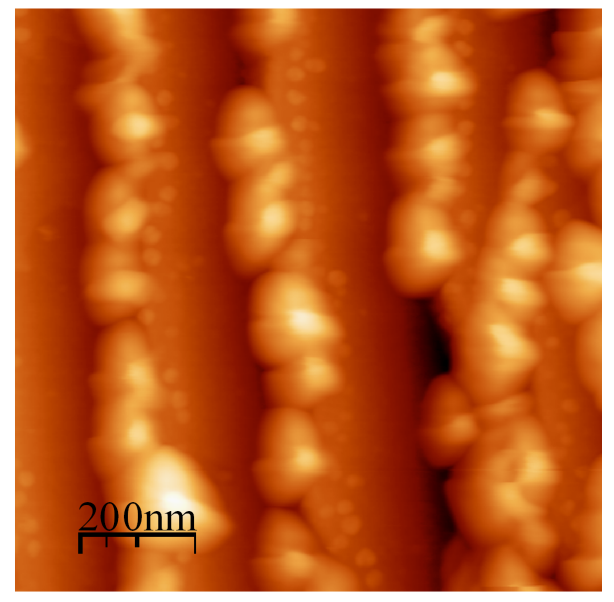

Fig. 2. The AFM image of Sb film of mean thickness equal to about $1.5 \mathrm{~nm}$ after a few scans; scanning area: $1 \times 1 \mu \mathrm{m}^{2}$. An accumulation of $\mathrm{Sb}$ on the steps of substrate's terraces is visible. Range of the color scale amounts from 0 to $50 \mathrm{~nm}$.

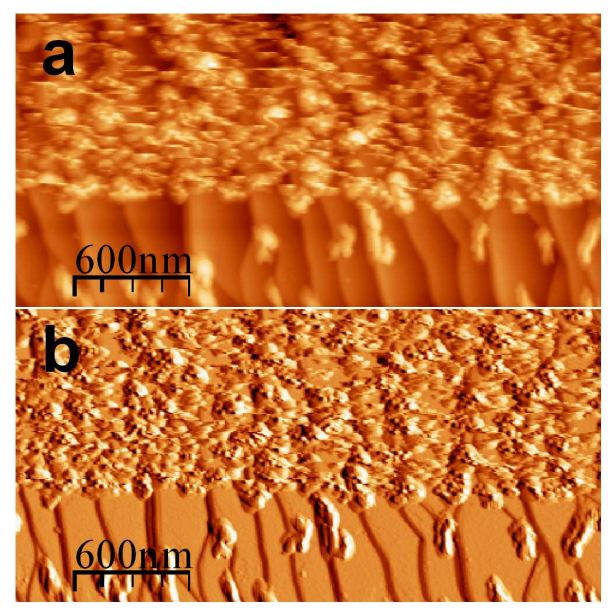

Fig. 3. (a) The AFM topography and the deflection image (b) showing the borderline between two regions of different degree of $\mathrm{Sb}$ film modification. The lower area was scanned three times, upper area only one time. The film is about $1.5 \mathrm{~nm}$ thick on average. Scanning area equals to $1 \times 0.5 \mu \mathrm{m}^{2}$. 


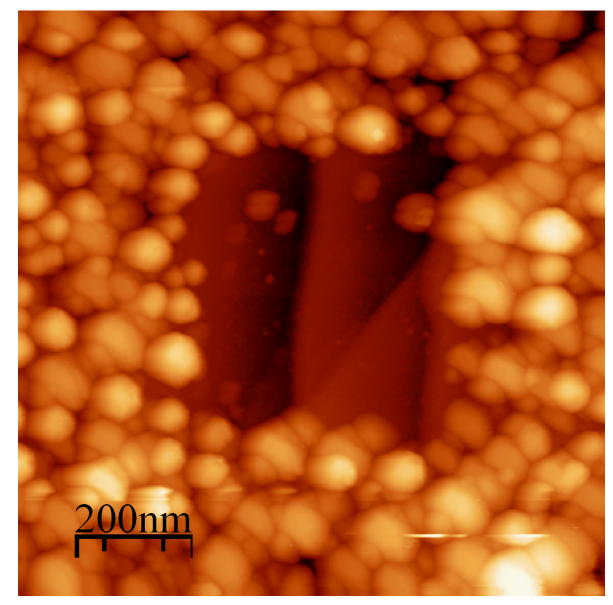

Fig. 4. The STM topography of about $25 \mathrm{~nm}$ thick Sb film modified by electric field applied to and/or current flowing through grains constituting the film. Scanning area equals to $1 \times 1 \mu \mathrm{m}^{2}$. The color scale range amounts from 0 to $50 \mathrm{~nm}$. Clean substrate surface of $400 \times 400 \mathrm{~nm}^{2}$ in size visible in the center of the micrograph is produced by the modification.

In fact, the $\mathrm{Sb} / \mathrm{SiC}(0001)$ system appears very difficult to be AFM imaged in contact mode if the Sb film thickness does not exceed $3 \mathrm{~nm}$. But, as it has been proved above, such films can be easily modified by this technique. Due to coalescence of the islands the thicker Sb layers become firm and therefore accessible to AFM imaging.

Coalesced Sb films completely shade the substrate surface. They consist of grains with diameter ranging from 60 to $140 \mathrm{~nm}$. Such layers cannot be modified by AFM, though they can be modified by the STM tip. Disintegration of the $\mathrm{Sb}$ grains is observed during the STM measurement when the tip bias exceeded $3.5 \mathrm{~V}$ (independently of the sign). The effect is very strong for positive and rather weak for negative bias voltages. As a result of the $\mathrm{Sb}$ grains disintegration the substrate surface is completely uncovered. An example of such modification is presented in Fig. 4. The modified area in the center of the STM micrograph reveals the substrate surface that is free of $\mathrm{Sb}$ and shows the typical terraced structure. Detailed mechanism of the disintegration of the Sb grains requires further studies. Most probably the grains disintegrate due to the field applied to and/or current flowing through them. The $\mathrm{Sb}$ atoms and molecules, which are the product of the disintegration, diffuse and support the growth of grains surrounding the modified area of the film.

\section{Conclusions}

We have proved that thin Sb films could be used for performing the SPM engineering on the nanoscale on $6 \mathrm{H}-$ $\mathrm{SiC}(0001)$ surface. Modification of ultrathin Sb films (up to $3 \mathrm{~nm}$ ) can be carried out by AFM. Thicker films (up to $45 \mathrm{~nm}$ ) can be modified by STM. Final result of the modifications is adsorbate free $6 \mathrm{H}-\mathrm{SiC}(0001)$ surface restitution over the modified area.

\section{Acknowledgments}

The authors would like to thank Dr. Stanisław Surma for helpful discussions and his assistance during preparation of manuscript. The work was supported by the University of Wrocław under the Grant 1010/S/IFD/14.

\section{References}

[1] X.N. Xie, H.J. Chung, C.H. Sow, A.T.S. Wee, Mater. Sci. Eng. $R$ 54, 1 (2006).

[2] D. Wouters, U.S. Angew, Chem. Int. Ed. 43, 2480 (2004).

[3] R. Garcia, R.V. Martinez, J. Martinez, Chem. Soc. Rev. 35, 29 (2006).

[4] A.A. Tseng, A. Notargiacomo, T.P. Chen, J. Vac. Sci. Technol. B 23, 877 (2005).

[5] Yu. Goldberg, M.E.Levinshtein, S.L. Rumyantsev in: Properties of Advanced Semiconductor Materials $G a N, A l N, S i C, B N, S i C, S i G e$, Eds. M.E. Levinshtein, S.L. Rumyantsev, M.S. Shur, Wiley, New York 2001, p. 93.

[6] N.G. Wright, A.B. Horsfall, K. Vassilevski, Mater. Today 11, 16 (2008).

[7] A.A. Gokhale, T.F. Kuech, M. Mavrikakis, J. Cryst. Growth 285, 146 (2005).

[8] I. Horcas R. Fernández, J.M. Gómez-Rodríguez, J. Colchero, J. Gómez-Herrero, A.M. Baro, Rev. Sci. Instrum. 78, 13705 (2007). 